\title{
Group B Streptococcal Renal Abscess in a 17-Year-Old Girl with Type 1 Diabetes Mellitus
}

\author{
Kyeong Eun Oh, M.D. \\ Hyung Eun Yim, M.D., Ph.D. \\ Kee Hwan Yoo, M.D., Ph.D. \\ Department of Pediatrics, Korea \\ University College of Medicine, Seoul, \\ Korea
}

Corresponding author:

Hyung Eun Yim, MD, PhD

Department of Pediatrics, Korea University

Ansan Hospital, Korea University College

of Medicine, 123 Jeokgeum-ro, Danwon-

gu, Ansan 15355, Republic of Korea

Tel: +82-31-412-5096

Fax: +82-31-405-8591

E-mail: he-yim@hanmail.net

Received: 5 March 2020

Revised: 27 March 2020

Accepted: 3 April 2020
This is an open-access article distributed under the terms of the Creative Commons Attribution Non-Commercial License (http:// creativecommons.org/licenses/by-nc/4.0/) which permits unrestricted non-commercial use, distribution, and reproduction in any medium, provided the original work is properly cited.

Copyright (C) 2020 The Korean Society of Pediatric Nephrology
Streptococcus agalactiae or group B streptococcus (GBS) is associated with infections in neonates and pregnant women. Herein, we describe a rare case of GBS renal abscess with peritonitis and pleural effusion in a 17-year-old girl with type 1 diabetes mellitus. The girl was admitted due to fever and right flank pain. Laboratory findings included leukocytosis and increased C-reactive protein level and erythrocyte sedimentation rate. Her serum glucose level was $484 \mathrm{mg} / \mathrm{dL}$. Urinalysis showed no pyuria. Renal sonography revealed parenchymal swelling in the right kidney. The patient was administered intravenous cefotaxime. Urine and blood cultures were negative. Fever seemed to improve, but the following day, she complained of abdominal pain and fever. Antibiotic was switched to imipenem, and abdominal and pelvic $\mathrm{CT}$ revealed a ruptured right renal abscess, peritonitis, and bilateral pleural effusion with atelectasis. Pigtail catheter drainage of the abscess was performed. Culture from the abscess was positive for GBS, and fever subsided 2 days after the drainage. She was discharged with oral cefixime. The clinical course of urinary tract infections (UTIs) can be atypical in patients with diabetes, and GBS can be a cause of UTIs. Prompt diagnosis and management are necessary to prevent complications in patients showing atypical courses.

Key words: Streptococcus agalactiae, diabetes mellitus, urinary tract infection, pleural effusion

\section{Introduction}

Streptococcus agalactiae or group B streptococcus (GBS) is a Gram-positive pathogen that is typically associated with infections in neonates and pregnant women ${ }^{1)}$. This organism is responsible for about $2-3 \%$ of all urinary tract infections (UTIs), and only a few cases of GBS renal abscesses have been reported

${ }^{2,3)}$. Moreover, diabetes mellitus (DM) is a major risk factor for complicated UTIs and serious GBS infections ${ }^{4,5}$. Herein, we describe a rare case of GBS renal abscess accompanied by peritonitis and bilateral pleural effusion in a 17-year-old girl with uncontrolled type $1 \mathrm{DM}$.

\section{Case report}

A 17-year-old girl was admitted due to fever and flank pain. She had been diagnosed with type I DM 3 years prior, but had stopped taking insulin and 
self-monitoring her blood sugar levels. She had experienced 2 days of fever and right flank pain without other urinary symptoms. Her body temperature was $38.8^{\circ} \mathrm{C}$, and her blood pressure was 120/70 mmHg. Right costovertebral angle tenderness was noted. The laboratory findings included leukocytosis (white blood cell count of $16,980 / \mu \mathrm{L}$, neutrophil-dominant) and an elevated C-reactive protein level and erythrocyte sedimentation rate $(15.4 \mathrm{mg} / \mathrm{dL}$ and $58 \mathrm{~mm} /$ $\mathrm{hr}$, respectively). The patient's plasma and urine neutrophil gelatinase-associated lipocalin levels were within the normal range, but her serum glucose level was $484 \mathrm{mg} / \mathrm{dL}$, and hemoglobin A1c level, 14.6\%. Urinalysis showed a $\mathrm{pH}$ of 5.5, a specific gravity of 1.040, glucosuria and ketonuria but no proteinuria, hematuria, or pyuria. Blood gas analysis showed a $\mathrm{pH}$ of $7.44, \mathrm{pCO}_{2}$ of $26 \mathrm{mmHg}$, and $\mathrm{HCO}_{3}{ }^{-}$of $17.8 \mathrm{mmol} /$ L. Initial renal sonography revealed diffuse wall thickening of the urinary bladder and hyperechoic parenchymal swelling in the right kidney. A dimercaptosuccinic acid renal scan was performed and revealed cortical defects in the right kidney. She was administered intravenous cefotaxime and insulin. The initial urine and blood cultures showed negative results. The patient's high fever and flank pain appeared to improve after treatment was initiated, and fever was not noted on hospital day 6. On the following day, however, she once again complained of abdominal pain and fever. No pyuria or hematuria was found on the urinalysis.

White blood cell count was decreased from $16,980 / \mu \mathrm{L}$ to $6,090 / \mu \mathrm{L}$ and C-reactive protein level was also decreased from $15.38 \mathrm{mg} / \mathrm{dL}$ to $2.87 \mathrm{mg} / \mathrm{dL}$. By contrast, erythrocyte sedimentation rate level was increased from $58 \mathrm{~mm} / \mathrm{hr}$ to $87 \mathrm{~mm} / \mathrm{hr}$.

Antibiotic resistance was suspected, so her antibiotic was switched to imipenem on hospital day 7. Abdominal and pelvic computed tomography (APCT) revealed a ruptured right renal and perinephric abscess, diffuse peritoneal thickening, ascites, and bilateral pleural effusion with passive atelectasis in basal segment of both lungs (Fig. 1A). On hospital day 8 , pigtail catheter drainage was performed and pus taken from the abscess was cultured (Fig. 1B). The abscess culture was positive for GBS. GBS was sensitive penicillin, cefotaxime, and imipenem. We maintained imipenem and the patient's fever subsided 2 days after the drainage of the abscess. Follow up sonography demonstrated no abscess pocket and tubogram of the pigtail catheter revealed no remaining connection to the vascular system (Fig. 1C, Fig. 2A). On hospital day 18, she was discharged with oral cefixime and subcutaneous insulin regimens. One month later, APCT showed improvement of the right renal abscess with cortical scarring at the lower pole of the right kidney (Fig. 2B).

\section{Discussion}

This is a rare case of a GBS renal abscess accompanied by peritonitis and bilateral pleural effusion in a 17-year-old girl with uncontrolled type 1 DM. Although she showed

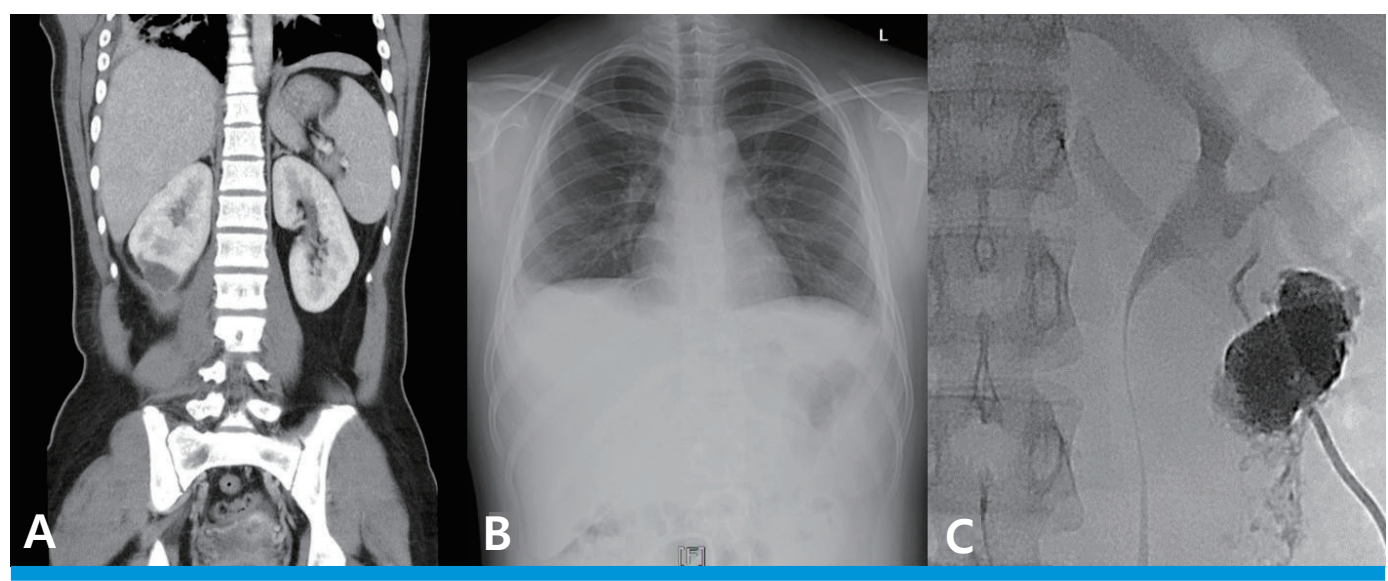

Fig. 1. Imaging studies demonstrating a ruptured right renal abscess with peritonitis, pleural effusion and catheter drainage of the abscess. (A) Abdomen pelvis CT demonstrating a ruptured right renal abscess, peritonitis, and bilateral pleural effusion with passive atelectasis in both basal lungs. (B) Chest X-ray showing bilateral pleural effusion. (C) Pigtail catheter drainage of the right renal abscess. 
initial clinical improvement upon intravenous administration of a third generation cephalosporin, her condition then deteriorated and APCT revealed a ruptured renal abscess with peritonitis and bilateral pleural effusion. After changing to a broad-spectrum $\beta$-lactam antibiotic and performing pigtail catheter drainage, the patient's fever subsided and she was discharged from the hospital.

GBS is common cause of perinatal infections in neonates and pregnant women ${ }^{1)}$, and a few cases of GBS-associated UTIs or renal abscesses have been reported ${ }^{3)}$. DM is known to be a major risk factor for serious GBS infections ${ }^{5)}$. The most common sites of infection are the skin, soft tissue, bones, joints, urinary tract, lungs, peritoneum, and genital tract $^{6}$. Patients with DM are also vulnerable to UTIs because the antimicrobial defense mechanisms of the kidney are insulin-dependent. Moreover, high glucose concentrations in the urine of DM patients may promote the growth of pathogenic bacteria ${ }^{7)}$. Therefore, patients with DM are at a higher risk of developing serious UTIs ${ }^{8)}$. In many studies, Escherichia coli was shown to be the most common pathogen responsible for UTIs among diabetic patients. One study described gram-negative bacteria such as Klebsiella, Proteus, and Pseudomonas also commonly cause UTIs in diabetic patients ${ }^{9)}$. On the other hand, another study reported that less common organisms such as coagulase-negative Staphylococci and Enterococcus species can cause UTIs in diabetic patients and showed that the multi-drug resistance rate was higher in diabetic patients with UTI than in patients without underlying $\mathrm{DM}^{10)}$. Our patient was a 17-year-old girl with DM, and GBS was found to be a pathogen underlying the complicated UTI. Her uncontrolled blood sugar levels may have increased glucose concentrations in the urine and impaired antimicrobial defense of her urinary system, leading to the development of serious renal infection.

Our patient presented with fever and costovertebral angle tenderness, but the initial urinalysis and urine culture revealed negative results. She didn't take any medicine including antibiotics before she came to our hospital. She was administered intravenous cefotaxime to treat the UTI, but redeveloped fever. APCT was reperformed and revealed a ruptured right renal abscess, peritonitis, and bilateral pleural effusion with passive atelectasis in both basal lungs. Why were the initial urinalysis and urine culture negative in this patient? One study reported that $25-30 \%$ of women with UTI symptoms have a negative urine culture because the infection does not affect the collecting system or involve obstruction of the collecting system ${ }^{11)}$. Moreover, in a small randomized controlled trial involving women with UTI symptoms but a negative dipstick urine test result, antibiotic treatment significantly reduced the median time to dysuria resolution compared to placebo ${ }^{12)}$. In our patient,

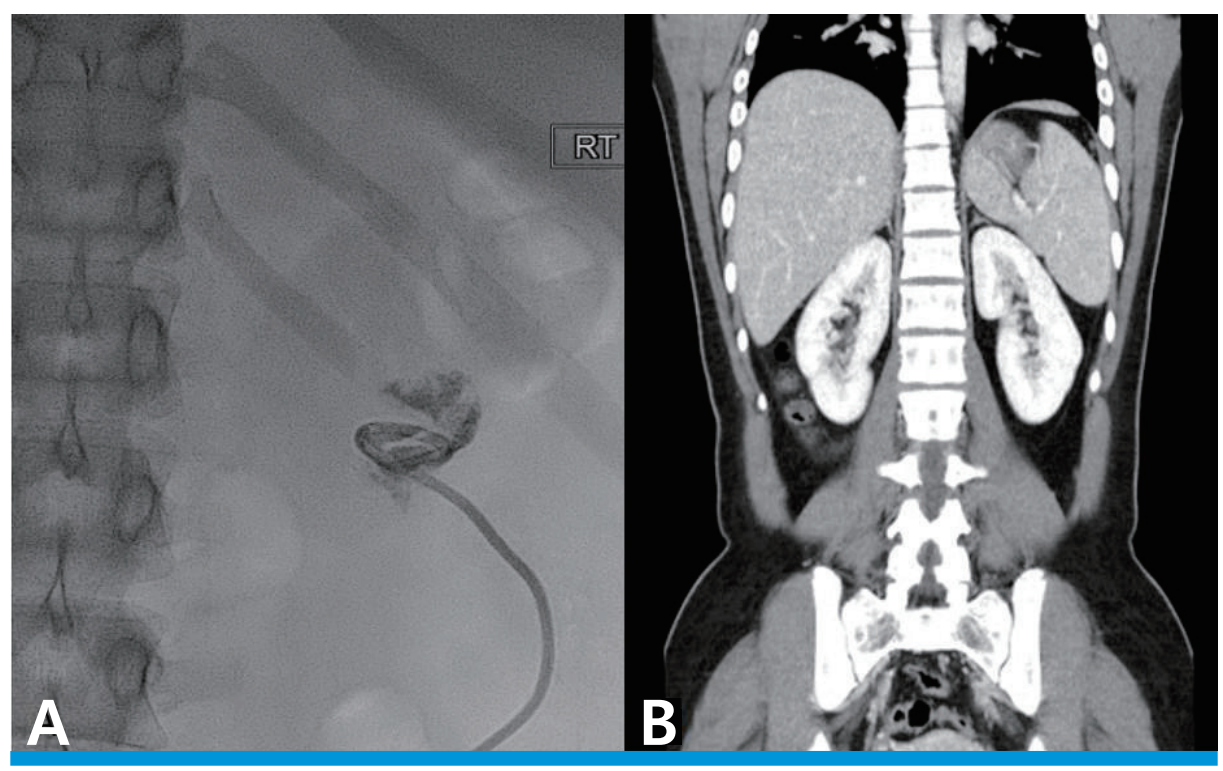

Fig. 2 Follow up imaging studies after treatment. (A) Tubogram with pigtail catheter demonstrating no link between the abscess pocket and the vascular system. (B) Follow up abdomen pelvis CT after 1 month showing improved right renal abscess with cortical scarring at right kidney lower pole. 
abscess did not seem to have communication with the renal collecting system and the location of the renal abscess. Empiric antibiotic treatment was initially effective, but catheter drainage and administration of antibiotic were ultimately required to successfully treat the ruptured renal abscess. In general, GBS is sensitive to penicillin and GBS that was detected in our patient was also sensitive to penicillin. We maintained imipenem to treat renal and perinephric abscess with diffuse peritonitis and bilateral pleural effusion effectively. There have been no cases of GBS renal abscess in children reported domestically or abroad. We found 1 case of GBS perinephric abscess in a 24-year-old woman with DM who experienced recurrent fever, flank pain, and increased urinary frequency for 1 month. Ultimately, she underwent percutaneous abscess drainage and was administered intravenous antibiotics to treat the GBS infection ${ }^{3)}$. Therefore, GBS infection should be considered in patients with underlying diseases such as diabetes who exhibit atypical UTI manifestations.

A variety of intra-abdominal or pelvic disorders such as renal and/or perirenal abscesses and urinary tract obstruction are associated with pleural effusion ${ }^{13)}$. A case of pleural empyema and perinephric abscess was recently reported in a patient who presented with a large right-sided pleural effusion $^{14)}$. In our case, bilateral pleural effusion and passive lung atelectasis were also noted alongside the ruptured renal abscess and peritonitis. The effusions were initially suspected to be of bacterial or tuberculous origin, but were ultimately deemed to be reactive effusions when the patient's clinical course was considered. Right-sided diffuse peritoneal thickening and pleural effusion were noted, so it is possible that the right perinephric abscess may have extended into the pleural cavity. Because the amount of effusion was relatively small, we did not perform diagnostic or therapeutic thoracentesis. The pleural effusion and renal abscess improved concurrently. No pleural or peritoneal complications were noted following treatment. Here, we chose to report this case because GBS renal and perinephric abscess are rare in children and adolescents, and the severity of the disease was such that it was accompanied by peritonitis and pleural effusion.

In conclusion, renal abscesses are relatively uncommon in children but may need a prolonged antibiotic treatment, an increased length of hospital stay, and a high treatment cost, and may be associated with life-threatening complications ${ }^{15)}$. The clinical course of UTIs can be atypical in patients with diabetes, and GBS can be a relevant organism causing renal abscess. Prompt diagnosis and management are necessary to prevent complications in UTI patients showing atypical clinical courses.

\section{Patient consent}

This study was approved by the institutional review board (IRB), and informed consent was waived due to retrospective study design and anonymous patient data2018AS0122).

\section{Conflicts of interest}

No potential conflict of interest relevant to this article was reported.

\section{References}

1. Ulett KB, Shuemaker JH, Benjamin WH, Tan CK, Ulett GC. Group B streptococcus cystitis presenting in a diabetic patient with a massive abdominopelvic abscess. J Med Case Rep 2012;6:237.

2. Leclercq SY, Sullivan MJ, Ipe DS, Smith JP, Cripps AW, Ulett GC. Pathogenesis of Streptococcus urinary tract infection depends on bacterial strain and B-hemolysin/cytolysin that mediates cytotoxicity, cytokine synthesis, inflammation and virulence. Nature 2012;6:29000.

3. Yoon KH, Lee JH, Han WJ, Cho MS, Kim JY, Park YS, et al. A perinephric abscess caused by Streptococcus agalactiae. Korean J Med 2009;76:220-3.

4. Mnif MF, Kamoun M, Kacem FH, Bouaziz Z, Charfi N, Mnif F, et al. Complicated urinary tract infections associated with diabetes mellitus: Pathogenesis, diagnosis and management. Indian J Endocrinol Metab 2013;17:442-5.

5. Yanai H, Hamasaki H, Tsuda N, Adachi H, Yoshikawa R, Moriyama S, et al. Group B streptococcus infection and diabetes: A review. J Microbiol Antimicrob 2012;4:1-5.

6. Batista RP, Ferreira CR. Streptococcus agalactiae septicemia in a patient with diabetes and hepatic cirrhosis. Autops Case Rep 2015;5:35-43.

7. Fünfstück R, Nicolle LE, Markolf H, Naber K. Urinary tract infection in patients with diabetes mellitus. Clin Nephrol 2012;77:40-8.

8. Zasloff M. Why are diabetics prone to kidney infections? J Clin 
www.chikd.org

Invest 2018;128:5213-5.

9. Hakeem LM, Bhattacharyya DN, Lafong C, Janjua KS, Serhan JT, Campbell IW. Diversity and complexity of urinary tract infection in diabetes mellitus. Br J Diabetes Vasc Dis 2009:9:119-25.

10. Woldemariam HK, Geleta DA, Tulu KD, Aber NA, Legese MH, Fenta GM, et al. Common uropathogens and their antibiotic susceptibility pattern among diabetic patients. BMC Infect Dis 2019;19:43.

11. Heytens S, De Sutter A, Coorevits L, Cools P, Boelens J, Van Simaey $L$, et al. Women with symptoms of a urinary tract infection but a negative urine culture: PCR-based quantification of Escherichia coli suggests infection in most cases. Clin Microbiol Infect 2017; 23:647-52.

12. Mangin D, Toop L, Chambers S, Fletcher L. Response to antibiotics of women with symptoms of urinary tract infection but negative dipstick urine test results: double blind randomised controlled trial. BMJ 2005;331:143.

13. Wang IK, Chuang FR, Chang HY, Lin CL, Yang CT. Acute pyelonephritis associated with transudative pleural effusion in a middleaged woman without urinary tract obstruction. Med Princ Pract 2006;15:309-11.

14. Tan PSC, Badiei A, Fitzgerald DB, Kuok YJ, Lee YCG. Pleural empyema in a patient with a perinephric abscess and diaphragmatic defect. Respirol Case Rep 2019;7:e00400.

15. Chen CY, Kuo HT, Chang YJ, Wu KH, Yang WC, Wu HP. Clinical assessment of children with renal abscesses presenting to the pediatric emergency department. BMC Pediatrics 2016;16:189. 